\title{
Maximum independent sets near the upper bound
}

\author{
Ingo Schiermeyer \\ Institut für Diskrete Mathematik und Algebra \\ Technische Universität Bergakademie Freiberg \\ 09596 Freiberg, Germany \\ Ingo.Schiermeyer@tu-freiberg.de
}

October 2, 2018

\begin{abstract}
The size of a largest independent set of vertices in a given graph $G$ is denoted by $\alpha(G)$ and is called its independence number (or stability number). Given a graph $G$ and an integer $K$, it is NP-complete to decide whether $\alpha(G) \geq K$. An upper bound for the independence number $\alpha(G)$ of a given graph $G$ with $n$ vertices and $m$ edges is given by $\alpha(G) \leq p:=\left\lfloor\frac{1}{2}+\sqrt{\frac{1}{4}+n^{2}-n-2 m}\right\rfloor$.

In this paper we will consider maximum independent sets near this upper bound. Our main result is the following: There exists an algorithm with time complexity $O\left(n^{2}\right)$ that, given as an input a graph $G$ with $n$ vertices, $m$ edges, $p:=\left\lfloor\frac{1}{2}+\sqrt{\frac{1}{4}+n^{2}-n-2 m}\right\rfloor$, and an integer $k \geq 0$ with $p \geq 2 k+1$, returns an induced subgraph $G_{p, k}$ of $G$ with $n_{0} \leq p+2 k+1$ vertices such that $\alpha(G) \leq p-k$ if and only if $\alpha\left(G_{p, k}\right) \leq p-k$. Furthermore, we will show that we can decide in time $O\left(1.2738^{3 k}+k n\right)$ whether $\alpha\left(G_{p, k}\right) \leq p-k$.
\end{abstract}

\section{Introduction}

We use [12] for terminology and notation not defined here and consider finite and simple graphs only. Given two graphs $G$ and $H$, then $G \cup H$ and $G+H$ denote the union and the join of $G$ and $H$, respectively.

An independent set (also called stable set) in a graph $G$ is a subset of pairwise nonadjacent vertices. The size of a largest independent set of vertices in a given graph $G$ is denoted by $\alpha(G)$ and is called its independence number (or stability number). The MAXIMUM InDEPENDENT SET problem (MIS for short) asks for finding an independent set of maximum cardinality in a graph. This problem is known to be NP-hard in general. Moreover, it remains NP-hard under several restrictions, for example for triangle-free graphs [8], $K_{1,4}$-free graphs [7], planar graphs of maximum degree at most three [5], and for sparse or dense graphs [10]. On the other hand, for some subclasses of these graph classes the MIS problem is solvable in polynomial time, for example for graphs with maximum degree two (folklore), for subclasses of subcubic graphs [2], and for $K_{1,3}$-free graphs [7, 9]. 


\section{Algorithmic bounds for the independence number of a graph}

Various lower and upper bounds for the independence number of a graph in terms of other graph parameters have been shown in the last decades. A survey on these bounds is given in [6], where the number of lower bounds is considerably larger than the number of upper bounds.

Recently, Dvořák and Lidický have considered maximum independent sets near the lower bound in a graph, where the lower bound is $\frac{n}{\Delta}$.

As mentioned in [4], by Brook's Theorem [1], every graph of order $n$, maximum degree at most $\Delta \geq 3$ and clique number at most $\Delta$ is $\Delta$-colourable, and thus has an independent set of size at least $\frac{n}{\Delta}$.

Theorem 1 (Dvořák and Lidický) There exists an algorithm with time complexity $O\left(\Delta^{2} n\right)$ that, given as an input an integer $\Delta \geq 3$, a graph $G$ of order $n$ with $\max (\Delta(G), \omega(G)) \leq \Delta$, and an integer $k \geq 0$, returns an induced subgraph $G_{0}$ of $G$ with $n_{0} \leq 114 \Delta^{3} k$ vertices such that $\alpha(G) \geq \frac{n}{\Delta}+k$ if and only if $\alpha\left(G_{0}\right) \geq \frac{n_{0}}{\Delta}+k$.

Hence the problem of deciding whether such a graph has an independent set of size at least $\frac{n}{\Delta}+k$ has a kernel of size $O(k)$. Note that these graphs belong to the class of sparse graphs. As mentioned in [4], such an instance can be solved by brute force leading to an $2^{O\left(\Delta^{3} k\right)}+O\left(\Delta^{2} n\right)$ algorithm.

In this paper we will consider independent sets near the upper bound in general graphs, where the upper bound is the following bound (cf. [6]).

Theorem 2 Let $G$ be a graph of order $n$ and size $m$. Then

$$
\alpha(G) \leq p:=\left\lfloor\frac{1}{2}+\sqrt{\left.\frac{1}{4}+n^{2}-n-2 m\right\rfloor} .\right.
$$

The main result in this paper is the following analogue of Theorem 1

Theorem 3 There exists an algorithm with time complexity $O\left(n^{2}\right)$ that, given as an input a graph $G$ of order $n$ with $p:=\left\lfloor\frac{1}{2}+\sqrt{\frac{1}{4}+n^{2}-n-2 m}\right\rfloor$, and an integer $k \geq 0$ with $p \geq 2 k+1$, returns an induced subgraph $G_{p, k}$ of $G$ with $n_{0} \leq p+2 k+1$ vertices such that $\alpha(G) \leq p-k$ if and only if $\alpha\left(G_{p, k}\right) \leq p-k$.

Furthermore, we will show that we can decide in time $O\left(1.2738^{3 k}+k n\right)$ whether $\alpha\left(G_{p, k}\right) \leq$ $p-k$. 


\section{Auxiliary results}

Given a graph $G$ of order $n$, a subset $U \subset V(G)$ of vertices is called a vertex cover of $G$, if for each edge of $G$ at least one of its incident vertices belongs to $U$. The following property is well-known:

$U$ is a vertex cover of $G$ if and only if $V(G) \backslash U$ is an independent set of $G$

Hence the problem of computing a minimum vertex cover is also NP-hard. However, given an integer $k \geq 1$, the decision problem whether $G$ has a vertex cover of size at most $k$ is fixed parameter tractable. This means, there exists an algorithm with time complexity $f(k) \cdot p(n)$, where $f(k)$ is a computable function depending only on $k$ and $p(n)$ is a polynomial depending on $n$. Several fpt-algorithms have been developed for the vertex cover problem, where the currently fastest algorithm is due to Chen et al. [3] with time complexity $O\left(1.2738^{k}+k n\right)$.

\section{Main results}

For a given a graph $G$ with $n$ vertices, $m$ edges, $p:=\left\lfloor\frac{1}{2}+\sqrt{\frac{1}{4}+n^{2}-n-2 m}\right\rfloor$, and an integer $k \geq 0$ with $p \geq 2 k+1$, let $H \subset G$ be the subgraph of $G$ induced by all vertices having degree at least $n-p+k$. Let $G_{p, k}=G-H$.

Theorem 4 There exists an algorithm with time complexity $O\left(n^{2}\right)$ that, given as an input a graph $G$ of order $n$ with $n$ vertices, $m$ edges, $p:=\left\lfloor\frac{1}{2}+\sqrt{\frac{1}{4}+n^{2}-n-2 m}\right\rfloor$, and an integer $k \geq 0$ with $p \geq 2 k+1$, returns an induced subgraph $G_{p, k}$ of $G$ with $n_{0} \leq p+2 k+1$ vertices such that $\alpha(G) \leq p-k$ if and only if $\alpha\left(G_{p, k}\right) \leq p-k$.

Proof: If $\alpha(G) \leq p-k$ then $\alpha\left(G_{p, k}\right) \leq p-k$ since $G_{p, k}$ is an induced subgraph of $G$. Conversely, suppose $\alpha\left(G_{p, k}\right) \leq p-k$, but $\alpha(G) \geq p-k+1$. Let $I$ be an independent set with $p-k+1$ vertices and $H \subset G$ be the subgraph of $G$ induced by all vertices having degree at least $n-p+k$. Then $I \cap V(H)=\emptyset$. Hence $I \subset G_{p, k}=G-H$. Then $d_{G}(v) \leq n-(p-k+1)=$ $n-p+k-1$ for all vertices in $G_{p, k}$. Using the handshaking lemma we obtain

$$
\frac{n_{0}}{2}(p-k)=\frac{n_{0}}{2}(n-1-(n-p+k-1)) \leq \frac{1}{2} \sum_{v \in V\left(G_{0}\right)}\left(n-1-d_{G}(v)\right)=m(\bar{G})<\left(\begin{array}{c}
p+1 \\
2
\end{array}\right)
$$

implying $n_{0}<\frac{p(p+1)}{p-k}$. Using $\frac{p(p+1)}{p-k}=p+\frac{p(k+1)}{p-k}$ we obtain $n_{0} \leq p+2 k+1$ for all $p \geq 2 k$.

Now we are searching for a vertex cover of size $t(k)=n_{0}-(p-k+1)$. For all $p \geq 2 k$ we obtain

$$
t(k)<\frac{p(p+1)}{p-k}-(p-k+1)=\frac{2 k p-k^{2}+k}{p-k}=2 k+\frac{k(k+1)}{p-k} \leq 3 k+1 .
$$

Hence $t(k) \leq 3 k$.

Using the vertex cover fpt-algorithm of Chen et al. we obtain the next theorem. 
Theorem 5 There exists an $O\left(1.2738^{3 k}+k n\right)$ fpt-algorithm (fixed parameter tractable) to decide whether $\alpha\left(G_{p, k}\right) \leq p-k$.

Corollary 1 Given as an input a graph $G$ with $n$ vertices, $m$ edges, $p:=\left\lfloor\frac{1}{2}+\sqrt{\frac{1}{4}+n^{2}-n-2 m}\right\rfloor$, and an integer $k \geq 0$ with $p \geq 2 k+1$, it can be decided in time $O\left(1.2738^{3 k}+k n\right)$ whether $\alpha(G) \leq p-k$.

Remark 1 Setting $p \geq c \cdot k$ for a constant $c>1$ we obtain $n_{0}<p+\frac{c}{c-1}(k+1)$ in Theorem 4. For the complexity we obtain $O\left(1.2738^{2 k-1+\left\lceil\frac{k+1}{c-1}\right\rceil}+k n\right)$.

\section{Improving the upper bound}

Before presenting an algorithm including our main results we will show an improvement of the upper bound using neighbourhood unions of pairs of nonadjacent vertices. This bound is easy computable and can eventually speed up the running time of the algorithm.

We start with the following upper bound for the independence number, which may be attributed as folklore. For a graph $G$ of order $n$ let $d_{1} \leq d_{2} \leq \ldots \leq d_{n}$ denote its degree sequence. Define $p_{1}(G):=\max \left\{i \mid d_{i} \leq n-i\right\}$. Note that $p_{1}(G)$ is well defined, since $d_{1} \leq n-1$. Then the following proposition holds.

Proposition 1 Let $G$ be a graph of order $n$ and degree sequence $d_{1} \leq d_{2} \leq \ldots \leq d_{n}$. Then

$$
\alpha(G) \leq p_{1}(G)
$$

A short proof can be deduced from the following Theorem of Welsh and Powell.

Theorem 6 (Welsh and Powell)[11] Let $G$ be a graph of order $n$ and degree sequence $d_{1} \geq$ $d_{2} \geq \ldots \geq d_{n}$. Then

$$
\chi(G) \leq \max \left\{\min _{1 \leq i \leq n}\left\{i, d_{i}+1\right\} .\right.
$$

It is well known that $\alpha(G) \leq c c(G)=\chi(\bar{G})$ for every graph $G$, where $c c(G)$ denotes the clique covering number of a graph $G$. Now applying Theorem 6 on $\bar{G}$ we obtain $\alpha(G) \leq$ $\max \left\{i \mid d_{i} \leq n-i\right\}$.

Proposition 1 can be extended as follows.

Proposition 2 Let $G$ be a graph of order $n$ and degree sequence $d_{1} \leq d_{2} \leq \ldots \leq d_{n}$. Then

$$
\alpha(G) \leq p_{1}(G) \leq p(G) .
$$


Proof: Suppose $p_{1}=p_{1}(G)>p(G)=p$. Then $\sum_{i=1}^{p_{1}}\left(n-1-d_{i}\right) \geq \sum_{i=1}^{p_{1}}\left(n-1-d_{p_{1}}\right) \geq$ $p_{1}\left(p_{1}-1\right) \geq(p+1) p>\bar{m}(G)$, a contradiction.

For a vertex $u \in V(G)$ and all vertices $v \in \bar{N}(u)$, let $n_{2}(u) \leq n_{3}(u) \leq \ldots \leq n_{t}(u)$ denote the sequence of the cardinalities of the neighbourhood unions $|N(u) \cup N(v)|$ with $t=n-d(u)$. Define $p_{2}(G):=\max \left\{k \mid G\right.$ has at least $k$ vertices $v$ with $\left.n_{k}(v) \leq n-k\right\}$.

Proposition $3 \alpha(G) \leq p_{2}(G)$.

Proof: Let $I$ be a maximum independent set of vertices with $|I|=k$. Let $I=\left\{v_{1}, \ldots, v_{k}\right\}$. Then $\left|N\left(v_{i}\right) \cup N\left(v_{j}\right)\right| \leq n-k$ for all pairs of vertices $v_{i}, v_{j}$ with $1 \leq i<j \leq k$. Hence $n_{k}\left(v_{i}\right) \leq n-k$ for $1 \leq i \leq k$ implying $p_{2}(G) \geq k=\alpha(G)$.

Theorem 7 Let $G$ be a graph of order $n$ and degree sequence $d_{1} \leq d_{2} \leq \ldots \leq d_{n}$. Then

$$
\alpha(G) \leq p_{2}(G) \leq p_{1}(G) \leq p(G)
$$

Proof: Suppose $p_{2}(G)>p_{1}(G)$. Then $d_{p_{2}}>n-p_{2}$ by the definition of $p_{1}(G)$. Let $v_{1}, \ldots, v_{p_{2}}$ be $p_{2}(G)$ vertices with $n c_{p_{2}}\left(v_{i}\right) \leq n-p_{2}$. Then $d\left(v_{i}\right) \leq n-p_{2}$ for $1 \leq i \leq p_{2}$ implying $d_{p_{2}} \leq n-p_{2}$, a contradiction. This shows that $p_{2}(G) \leq p_{1}(G)$. Now the result follows with Proposition 2 and Proposition 3 .

Remark 2 For two integers $n, p$ with $n>p \geq 2$ let $H_{n, p}=K_{n-p}+p K_{1}$. Then $\alpha\left(H_{n, p}\right)=$ $p_{2}\left(H_{n, p}\right)=p_{1}\left(H_{n, p}\right)=p\left(H_{n, p}\right)$. This shows that there are infintely many graphs $G$ with $\alpha(G)=p_{2}(G)=p_{1}(G)=p(G)$.

\section{ALGORITHM}

1. For a graph $G$ with $n$ vertices and $m$ edges compute $p=p(G)$ and $p_{1}(G)$.

2. If $p_{1}(G) \leq p-k$, then Output: YES

3. Else compute $p_{2}(G)$.

4. If $p_{2}(G) \leq p-k$, then Output: YES

5. Else compute $G_{p, k}$ and decide whether $\alpha\left(G_{p, k}\right) \leq p-k$ using the vertex cover fptalgorithm. 


\section{Extremal graphs}

In this section we will describe the structure of extremal graphs $G_{p, k}$.

For an independent set $I$ of a graph $G$ a set $S$ is called an augmenting set for $I$, if $(I \backslash N(S)) \cup S$ is an independent set with $|(I \backslash N(S)) \cup S|>|I|$.

Now let $I$ be a maximum independent set in $G_{0}$, and let $R=V\left(G_{0}\right) \backslash I$. Then for any $t$ independent vertices $x_{1}, \ldots, x_{t}$ in $R$ there are $t$ vertices $y_{1}, \ldots, y_{t}$ in $I$ such that $x_{i} y_{i} \in E(G)$ for $1 \leq i \leq t$.

Suppose, $\alpha(G) \leq p-(k-1)$ and we obtain the answer "YES" to the test $\alpha\left(G_{p, k}\right) \geq p-k+1$. Then $\alpha(G)=\alpha\left(G_{p, k}\right)=p-k+1$. Let $I$ be a maximum independent set in $G_{p, k}$, and set $V\left(G_{p, k}\right)=I \cup R$ with $r=|R|$. By the construction of $G_{p, k}$ we have $d_{\bar{G}}(v) \geq p-k$ for each vertex $v \in V\left(G_{p, k}\right)$. Then

$$
\left(\begin{array}{c}
p+1 \\
2
\end{array}\right)>m\left(\bar{G}_{p, k}\right) \geq|E(I)|+|E(I, R)|+|E(R)| .
$$

Let $E^{*}=E(I, R) \cup E(R)$. Then $\left|E^{*}\right|=|E(I, R)|+|E(R)| \leq\left(\begin{array}{c}p+1 \\ 2\end{array}\right)-1-\left(\begin{array}{c}p-k+1 \\ 2\end{array}\right)$. Hence $\left|E^{*}\right| \leq p-1$ for $k=1,\left|E^{*}\right| \leq 2 p-2$ for $k=2$, and $\left|E^{*}\right| \leq 3 p-4$ for $k=3$.

On the other hand, using the handshaking lemma,

$$
\left|E^{*}\right| \geq \max \left\{r(p-k)-\left(\begin{array}{l}
r \\
2
\end{array}\right), \frac{r(p-k)}{2}\right\} .
$$

This inequality has the following properties.

$$
\begin{aligned}
& r(p-k)-\left(\begin{array}{l}
r \\
2
\end{array}\right) \text { is increasing for } 1 \leq r \leq p-k . \\
& \frac{r(p-k)}{2} \geq(p-k+1) \frac{p-k}{2} \text { for } r \geq p-k+1 .
\end{aligned}
$$

Now the extremal graphs can be generated, if we require $p \geq p(k)$ for a given integer $k \geq 0$.

1. $\alpha(G)=p, k=1, p \geq 3$

We have $\frac{r(p-1)}{2} \leq\left|E^{*}\right| \leq p-1$, hence $r \leq 2$. By (P1) we have $2(p-1)-1=2 p-3>p-1$ for $r=2$ and $p \geq 3$. Hence we may assume $0 \leq r \leq 1$ and we distinguish these two cases. Let $I$ be a maximum independent set of size $|I|=p$. Then there are no augmenting sets for $I$. This leads to the following graph structures.

(a) $n_{0}=p, r=0$

Then $G_{p, 1} \cong p K_{1}$.

(b) $n_{0}=p+1, r=1$

Then $K_{2} \cup(p-1) K_{1} \subset G_{p, 1} \subset p K_{1}+K_{1}$. 
2. $\alpha(G)=p-1, k=2, p \geq 8$

We have $\frac{r(p-2)}{2} \leq\left|E^{*}\right| \leq 2 p-2$. If $r \geq p-k+1=p-1$, then by (P2) we obtain $\left|E^{*}\right| \geq(p-1) \frac{5}{2}>2 p-2$ for $p \geq 7$, a contradiction. Hence we have $r \leq p-2$. By (P1) we have $3(p-2)-3=3 p-9>2 p-2$ for $r=3$ and $p \geq 8$. Hence we may assume $0 \leq r \leq 2$ and we distinguish these three cases. Let $I$ be a maximum independent set of size $|I|=p-1$. Then there are no augmenting sets for $I$. This leads to the following graph structures.

(a) $n_{0}=p-1, r=0$

Then $G_{p, 2} \cong(p-1) K_{1}$.

(b) $n_{0}=p, r=1$

Then $K_{2} \cup(p-2) K_{1} \subset G_{p, 2} \subset(p-1) K_{1}+K_{1}$.

(c) $n_{0}=p+1, r=2$

Then $K_{3} \cup(p-2) K_{1} \subset G_{p, 2} \subset(p-1) K_{1}+K_{2}$

or $\quad 2 K_{2} \cup(p-3) K_{1} \subset G_{p, 2} \subset(p-1) K_{1}+K_{2}$

3. $\alpha(G)=p-2, k=3, p \geq 15$

We have $\frac{r(p-2)}{2} \leq\left|E^{*}\right| \leq 3 p-4$. If $r \geq p-k+1=p-2$, then by (P2) we obtain $\left|E^{*}\right| \geq(p-2) \frac{p-3}{2}>3 p-4$ for $p \geq 10$, a contradiction. Hence we have $r \leq p-3$. By (P1) we have $4(p-3)-6=4 p-18>3 p-4$ for $r=4$ and $p \geq 15$. Hence we may assume $0 \leq r \leq 3$ and we distinguish these four cases. Let $I$ be a maximum independent set of size $|I|=p-2$. Then there are no augmenting sets for $I$. This leads to the following graph structures.

(a) $n_{0}=p-2, r=0$

Then $G_{p, 3} \cong(p-2) K_{1}$.

(b) $n_{0}=p-1, r=1$

Then $K_{2} \cup(p-3) K_{1} \subset G_{p, 3} \subset(p-2) K_{1}+K_{1}$.

(c) $n_{0}=p, r=2$

Then $K_{3} \cup(p-3) K_{1} \subset G_{p, 3} \subset(p-2) K_{1}+K_{2}$

or $\quad 2 K_{2} \cup(p-4) K_{1} \subset G_{p, 3} \subset(p-2) K_{1}+K_{2}$

(d) $n_{0}=p+1, r=3$

Then $K_{4} \cup(p-3) K_{1} \subset G_{p, 3} \subset(p-2) K_{1}+K_{3}$

or $\quad K_{3} \cup K_{2} \cup(p-4) K_{1} \subset G_{p, 3} \subset(p-2) K_{1}+K_{3}$

or $\quad 3 K_{2} \cup(p-5) K_{1} \subset G_{p, 3} \subset(p-2) K_{1}+K_{3}$ 


\section{References}

[1] R. L. Brooks, On colouring the nodes of a network, In: mathematical Proceedings of the Cambridge Philosophical Society, volume 37 (1941) 194-197.

[2] C. Brause, N. Lê, and I. Schiermeyer, The Maximum Independent Set Problem in Subclasses of Subcubic Graphs, Discrete Math. 338 (10) (2015) 1766-1778.

[3] J. Chen, I. Kanj, and G. Xia, Improved upper bounds for vertex cover, Theoret. Computer Science 411 (4042) (2010) 3736-3756.

[4] Z. Dvořák and B. Lidický, Independent sets near the lower bound in bounded degree graphs, Proceedings STACS 2017, arXiv:1609.09134v2 [cs.DM].

[5] M. Garey and D. Johnson, The rectilinear Steiner tree problem is NP-complete, SIAM J. Appl. Math. 32 (1977) 826-834.

[6] C. E. Larson, The independence number project: $\alpha$-bounds, in: The Conjecturing Project, Virginia Commonwealth University, https://independencenumber.wordpress.com, 2017.

[7] G. Minty, On maximal independent sets of vertices in claw-free graphs, J. Combin. Theory B 28 (1980) 284-304.

[8] S. Poljak, A note on stable sets and colorings of graphs, Comment. Math. Univ. Carolinae 15 (1974) 307-309.

[9] N. Sbihi, Algorithme de recherche d'un stable de cardinalité maximum dans un graphe sans étoile, Disccrete Math. 29 (1980) 53-76.

[10] I. Schiermeyer, Problems remaining NP-complete for sparse or dense graphs, Discuss. Math. Graph Theory 15 (1995) 33-41.

[11] D. J. A. Welsh and M. B. Powell, An upper bound for the chromatic number of a graph and its application to timetabling problems, The Computer Journal, 10 (1) (1967) 8586, doi:10.1093/comjnl/10.1.85

[12] D. B. West, Introduction to Graph Theory, Prentice Hall, 2001. 